\title{
On injective profinite algebras
}

\section{B.J. Day}

It is shown that if $\mathcal{C}$ is a finitely productive and hereditary class of uniformly pointed finite discrete universal algebras then any injective in $\mathcal{C}$ is an injective in ProC.

\section{Introduction}

All notation will be as in Banaschewski [1]. In [1] it is shown that certain properties of $C$ are inherited by ProC. The corresponding question for injectives has been studied by Choe [2], where results for associative and distributive universal algebras are obtained.

The aim of this note is to give results on injectivity under different hypotheses to those used by Choe. Our results hold for classes of pointed universal algebras for which each regular epimorphism $f$ is the coequaliser of ker $f$ and 0 ; for example, groups, algebras over a ring, additive categories, and so on. Such a category will be said to be uniformly pointed.

The results are useful in Day [3]; see [3], Definition 2.1, and [3], Remark 2.2. For instance, if we have an object $I$ which is injective in $F A$, then the algebraic theory $C=\left\{1, I, I^{2}, \ldots, I^{n}, \ldots\right\} \subset F A$ is a "basic" set of injectives in Pro A; hence in $C^{\prime}=S(P C)$. This then leads to results on duality, as in [3].

The proof of the result is much simpler than Choe's (whenever the two coincide) and is borrowed from Kaplan [4], Theorem 1. For basic category theory we refer the reader to Mac Lane [5] and Schubert [6].

Received 6 September 1978. 


\section{Injectives in Proc}

Let $A$ be the category of algebras for a finitary algebraic theory, and suppose further that $A$ is uniformly pointed (as explained in the Introduction). Let $C$ be a finitely productive and hereditary class of finite discrete algebras, and let $\mathrm{ProC}$ be the cogenerated category of Proc algebras (as constructed by Banaschewski [1]).

THEOREM 1. An object $I \in \mathcal{C}$ is injective in $\mathrm{C}$ if and only if it is injective in Proc.

Proof. Suppose $I$ is injective in $C$ and let $f: A \rightarrow I$ be a continuous homomorphism from $A \in$ ProC. Also let $i: A \rightarrow G=\prod C_{\lambda}$ be a closed embedding into a product of $C_{\lambda} \in \mathcal{C}$. We show that there exists a lifting $f^{\prime}: G \rightarrow I$ of $f$ along $i$. Consider the following diagram:

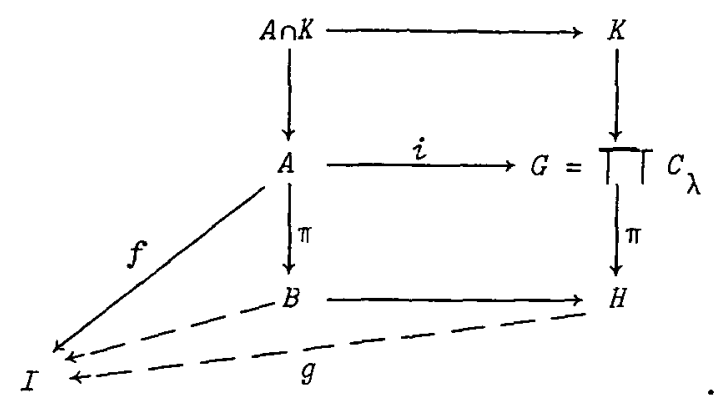

Here $f^{-1}(0)$ is open in $A$ and so contains a neighbourhood $A \cap V$ where $V=V_{\lambda_{1}} \times \ldots \times V_{\lambda_{n}} \times \prod_{\lambda \neq \lambda_{i}} c_{\lambda}, V_{\lambda_{i}}$ being a neighbourhood of 0 in $c_{\lambda_{i}}$. Set $H=C_{\lambda_{1}} \times \ldots \times c_{\lambda_{n}}$ and $K=\prod_{\lambda \neq \lambda_{i}} c_{\lambda}$. Then $f(A \cap K)=0$, since $f^{-1}(0) \supset A \cap K$; also $K$ is the kernel of $\pi$. Let $B=\operatorname{coker}(A \cap K)$. Then $B \rightarrow H$ is a monomorphism and $B \in \mathcal{C}$. But $f$ factors through $B$, so it lifts to $g$ by injectivity of $I$ in $C$. Then $g \pi$ is $f^{\prime}$, as required. $/ /$

COROLLARY 2. If $C$ has enougin injectives, then so has Proc, and every injective in ProC is a retract of a product of injectives from $C$.

Proof. Each $A \in$ Proc admits an embedding $A \rightarrow \prod c_{\lambda}, c_{\lambda} \in \mathcal{C}$. 
Thus $A$ is embedded in $\prod I_{\lambda}$, where each $C_{\lambda} \rightarrow I_{\lambda}$ is an injective extension in $C$. The result then follows from injectivity. //

\section{References}

[1] B. Banaschewski, "On profinite universal algebras", General topology and its relations to modern analysis and algebra III, 51-62 (Proc. 3rd Prague Topological Symposium, 1971. Academia Publishing House of the Czechoslovak Academy of Sciences, Prague; Academic Press, New York, London, 1972).

[2] Tae Ho Choe, "Injective and projective zero-dimensional compact universal algebras", Algebra Universalis 7 (1977), 137-142.

[3] B.J. Day, "Duality in topological algebra", Buzz. Austral. Math. Soc. 18 (1978), 475-480.

[4] Samuel Kaplan, "Extensions of the Pontryagin duality II: direct and inverse sequences", Duke Math. J. 17 (1950), 419-435.

[5] Saunders Mac Lane, Categories for the working mathematician (Graduate Texts in Mathematics, 5. Springer-Verlag, New York, Heidelberg, Berlin, 1971).

[6] Horst Schubert, Categories (translated by Eva Gray. Springer-Verlag, Berlin, Heidelberg, New York, 1972).

Department of Pure Mathematics,

University of Sydney,

Sydney,

New South Wales. 